This is the final peer-reviewed accepted manuscript of:

Federico Ferrarese Lupi*, Tommaso Jacopo Giammaria, Andrea Miti, Giampaolo Zuccheri, Stefano Carignano, Katia Sparnacci, Gabriele Seguini, Natascia De Leo, Luca Boarino, Michele Perego*, and Michele Laus*

Hierarchical Order in Dewetted Block Copolymer Thin Films on Chemically Patterned Surfaces

ACS Nano 2018, 12, 7, 7076-7085

The final published version is available online at: https://doi.org/10.1021/acsnano.8b02832

Rights / License:

The terms and conditions for the reuse of this version of the manuscript are specified in the publishing policy. For all terms of use and more information see the publisher's website. 


\title{
Hierarchical order in dewetted block copolymer thin films on chemically patterned surfaces
}

Federico Ferrarese Lupi,${ }^{1 *}$ Tommaso Jacopo Giammaria, ${ }^{2,3}$ Andrea Miti ${ }^{4}$ Giampaolo Zuccheri, ${ }^{4}$ Stefano Carignano, ${ }^{5}$ Katia Sparnacci, ${ }^{3}$ Gabriele Seguini, ${ }^{2}$ Natascia De Leo, ${ }^{1}$ Luca Boarino, ${ }^{1}$ Michele Perego, ${ }^{2}$ Michele Laus ${ }^{3}$

${ }^{1}$ Nanoscience and Materials Division, Istituto Nazionale di Ricerca Metrologica, Strada delle Cacce 91, 10135 Torino, Italy

${ }^{2}$ CNR-IMM, Unit of Agrate Brianza, Via C. Olivetti 2, 20864 Agrate Brianza, Italy

${ }^{3}$ Dipartimento di Scienze e Innovazione Tecnologica (DISIT), Università del Piemonte Orientale “'A. Avogadro", Viale T. Michel 11, 1512 Alessandria, Italy

${ }^{4}$ Dipartimento di Farmacia e Biotecnologie e Istituto di Nanoscienze del CNR (S3-Modena), Via Irnerio, 48 - 40126 BOLOGNA.

${ }^{5}$ LNGS-INFN, Via G. Acitelli 22, 67100 Assergi (AQ),Italy

Corresponding author e-mail: f.ferrareselupi@inrim.it

KEYWORDS: block copolymers, dewetting, self-assembly, nano droplets, rapid thermal processing

\begin{abstract}
We investigated the dewetting process on flat and chemically patterned surfaces of ultrathin films (thickness between 2 and $15 \mathrm{~nm}$ ) of a cylinder forming polystyrene-block-poly (methyl methylacrylate) (PSb-PMMA) spin coated on poly(styrene-r-methyl methylacrylate) random copolymers (RCP). When the PS-bPMMA film dewets on a $2 \mathrm{~nm}$ thick RCP layer, the ordering of the hexagonally packed PMMA cylinders in the dewetted structures extends over distances far exceeding the correlation length obtained in continuous Block Copolymer (BCP) films. As a result, micrometer size circular droplets featuring defectless single grains of self-assembled PS-b-PMMA with PMMA cylinders perpendicularly oriented with respect to the substrate, are generated and result randomly distributed on the substrate. Additionally, alignment of the droplets along micrometric lines was achieved by performing the dewetting process on large-scale chemical patterned stripes of $2 \mathrm{~nm}$ thick RCP films by laser lithography. By properly adjusting the periodicity of the chemical pattern, it was possible to tune and select the geometrical characteristics of the dewetted droplets in terms of maximum thickness, contact angle and diameter while maintaining the defectless single grain perpendicular cylinder morphology of the circular droplets.
\end{abstract}


The development of a technology to determine the size and registration of nanoscaled features represents a cutting-edge topic in material science. To address this issue, a wealth of different fabrication techniques based on self-assembling (SA) materials including liquid crystals, ${ }^{1,2}$ monodispersed nanosphere systems s.4 $^{3,4}$ and block copolymers $(\mathrm{BCP})^{5,6}$ have been introduced, developed and eventually integrated in advanced manufacturing processes. Among them, the combination of conventional lithographic techniques with BCP thin films has proven itself to be a versatile nanofabrication tool to produce well registered nanostructured polymeric masks with feature size below $20 \mathrm{~nm} \cdot{ }^{7,8}$ For microelectronics ${ }^{9,10}$ and metrology ${ }^{11,12}$ applications, most of the work was addressed to flat and homogeneous BCP films deposited and self-assembled on planar surfaces to prepare nanolithographic masks, i.e. using the BCP template as passive material for the subsequent processing of the substrate. In this context, several attempts to integrate BCP mask in etching, ${ }^{13,14}$ lift-off ${ }^{15,16}$ and sequential infiltration processes ${ }^{17,18}$ were reported. However, for a large number of alternative applications, BCPs are used as active nanostructured material. Well established examples are the fabrication of filtration membranes ${ }^{19-21}$ or photonics systems. ${ }^{22,23}$ For this type of applications, the processing of BCP on curved substrates ${ }^{24}$ or the generation of non-planar geometries ${ }^{25}$ is required.

A promising tool for creating three-dimensional SA patterns with curved shape is to induce dewetting in polymeric films. This method has been successfully adopted in thin homopolymeric films to control the density and aggregation of nanoparticles ${ }^{26,27}$, to enhance the photoluminescence of quantum dots ${ }^{28}$ and to fabricate circular nanolenses..$^{29,30}$ The foundation of these achievements arises from the vast literature concerning the understanding of the mechanisms ${ }^{31,32}$ and the control over morphology $y^{33-36}$ and position ${ }^{37,38}$ of dewetted homopolymer films. The same degree of control over dewetted BCP films would allow hierarchical nanostructures to be developed for advanced functional materials (e.g. plasmonics, ${ }^{39}$ superconductors, ${ }^{40}$ metamaterials, ${ }^{41}$ stretchable electronics ${ }^{42}$ or polymeric micelles ${ }^{43}$ ). Nevertheless, the understanding of dewetting phenomena in BCP thin films is not trivial due to the high complexity of the system. In fact, besides the contributions inherent to the film thickness (e.g. van der Waals forces and interfacial energy at the substrate and air interfaces) and external perturbations (e.g. substrate roughness, ${ }^{44}$ thermal fluctuations or mechanical vibrations), the BCP film instability depends also on the BCP self-assembly characteristics. ${ }^{45}$ These characteristics are connected to the relative volume fraction $f$ of the two blocks making up the BCP, the overall molecular weight of the macromolecule and the Flory-Huggins parameter.

Interestingly, in many cases, BCP film instability was investigated in the disordered state above the order-disorder transition temperature $\left(T_{O D T}\right)^{46,47}$, without considering the effect of the nano-scale ordering process inherent in these SA materials. As a matter of fact, very thin BCP films undergo reorganization processes at two distinct scales, micro- (i.e. dewetting) and nano-scale (i.e. orientation and ordering of the SA features), thus a comprehensive investigation should consider the reciprocal influence exerted by these two processes. In addition, the processing parameters (i.e. annealing rate, temperature and time, atmosphere inside the annealing chamber, characteristics of the thermal ramps) play an active role in the dewetting 
mechanism and kinetics ${ }^{48}$ simultaneously affecting the SA process. From another perspective, this interplay between dewetting and SA processes was implicitly targeted by analyzing BCP films deposited on physically patterned substrates, ${ }^{38}$ nanoimprinting ${ }^{49}$ or electro-hydrodynamic jet printing ${ }^{50,51}$ modified surfaces. However, in all these studies the main objective was the control over the position of the dewetted droplets, whereas the SA process was considered as a secondary aspect with no direct impact on the dewetting process.

Nevertheless there is a crucial factor impacting both SA and dewetting processes, namely the interaction between BCP film and substrate. ${ }^{52-54} \mathrm{~A}$ very efficient and widespread approach to control orientation and long-range ordering of SA BCP features consists in grafting a layer of a random copolymer (RCP) with tuned composition and molar mass to the substrate. ${ }^{55-58}$ Using this approach to modify the substrate surface, in a recent paper, we reported on the ordering dynamics of a cylinder forming polystyrene-block-poly(methyl methylacrylate) (PS- $b$-PMMA) BCP (styrene fraction $f=0.71, M_{n}=67.1 \mathrm{~kg} \cdot \mathrm{mol}^{-1}$ ) deposited on a poly(styrene-r-methyl methylacrylate) $(\mathrm{P}(\mathrm{S}-r$-MMA $))\left(f=0.61, M_{n}=14.5 \mathrm{~kg} \cdot \mathrm{mol}^{-1}\right) \mathrm{RCP}$ layer. We studied the BCP layer ordering as a function its thickness in the range from $250 \mathrm{~nm}$ to $10 \mathrm{~nm}$. However, the BCP film became unstable for thickness values $h_{B C P}<10 \mathrm{~nm} .{ }^{59}$ In the present paper, we investigate the influence of the substrate neutralization on the ordering of SA features on the morphological characteristic of dewetted PS-b-PMMA films pattern with layer thickness ranging from 2 to $15 \mathrm{~nm}$. The study is performed on substrates homogeneously neutralized by different P(S-r-MMA) RCP layers and on chemically patterned substrates, consisting of a periodic replication of stripes with different wetting behavior prepared by laser writer lithography.

\section{RESULTS AND DISCUSSION}

\section{Micro-scale dewetting}

BCP films with different thickness $2 \leq h_{B C P} \leq 15 \mathrm{~nm}$ were spin coated on substrates previously neutralized with two different P(S-r-MMA) RCPs with grafting thickness of 2 and $7.5 \mathrm{~nm}$ (named in this manuscript R2 and $\mathrm{R} 7$ respectively). Before the annealing treatment, all the BCP films present a continuous and flat surface without any sign of ruptures that can be ascribed to a dewetting occurring at room temperature during the spin coating process. On the other hand, after the thermal annealing performed in RTP at $250{ }^{\circ} \mathrm{C}$ for $300 \mathrm{~s}$, clear evidence of dewetting phenomenon is observed in very thin BCP films. Figure 1 reports the SEM micrographs of the surface morphology of the annealed BCP films with different $h_{B C P}$ deposited on R2 (Figures 1a-d) and R7 (Figures 1e-h). For completeness, SEM micrographs with higher magnification are also reported in S1 and S2. In general, for thick BCP films the surface is continuous irrespectively of the RCP thickness. However, it is possible to identify a limit thickness $\left(h_{s t}\right)$, below which the film becomes unstable and the dewetting process takes place. According to the definitions given by Seemann et al. ${ }^{33}$ the existence of a $h_{s t}$ classifies these systems, i.e. $\mathrm{BCP}$ deposited on $\mathrm{R} 2$ or $\mathrm{R} 7$, as metastable. To determine $h_{s t}$ while measuring at the same time 
the percentage of area covered by the $\mathrm{BCP}$ a complete analysis of the dewetting has been carried out by processing the SEM micrographs with a grain marking routine. ${ }^{60}$ When the software detected no grains, we assumed that the BCP covered $100 \%$ of the scanned area. In the case of the BCP on R2 the dewetting starts below $h_{s t}=14.5 \mathrm{~nm}$ while on R7 the BCP film is stable down to $h_{s t}=12 \mathrm{~nm}$ (Figure 1i). For $h_{B C P}<h_{s t}$ the BCP dewets following a morphology sequence consisting in micrometric holes in the films, bicontinuous structures and circular droplets as the film thickness decreases, in good agreement with literature observations for symmetric BCPs. ${ }^{61}$ Although the same morphology sequence is observed for both RCPs, in the two systems under consideration the appearance of the aforementioned morphologies occurs at different $h_{B C P}$.
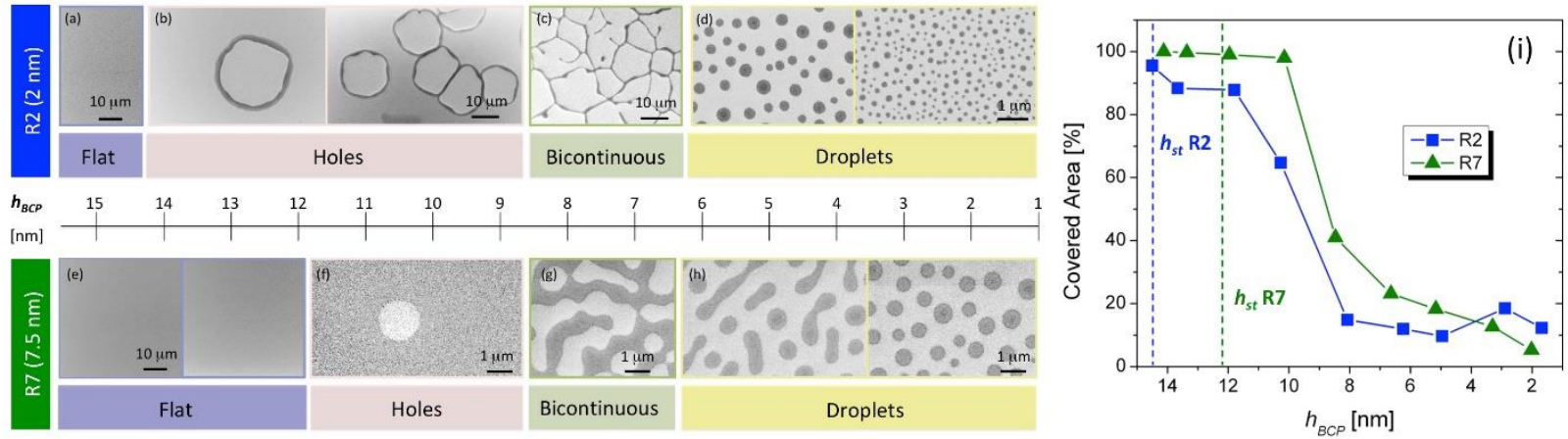

Figure 1: SEM micrographs describing the dewetting behavior as a function of the BCP thickness on R2 (a)-(d) and R2 (e)-(h). (i) Percentage of area covered by the BCP film after the thermal annealing process for both RCPs as a function of the deposited film thickness before the RTP annealing.

Below $h_{s t}$ the formation of circular holes in the BCP film is observed, with an average diameter dependent on the RCP. For R2, the size of the circular holes exceeds several tens of micrometers (Figure 1b) whereas for R7 the size is only one to a few micrometers (Figure 1f). As $h_{B C P}$ decreases below $8 \mathrm{~nm}$, the coalescence of circular holes leads to a sort of bicontinuous pattern consisting of long stripes connected one to each other (Figures 1c and $1 \mathrm{~g}$ ). This behavior is replicated in the thickness window $8 \mathrm{~nm}>h_{B C P}>6 \mathrm{~nm}$ for both RCPs. Finally, below $6 \mathrm{~nm}$ small droplets are formed with elliptical or circular shape (see Figures $1 \mathrm{~d}$ and $1 \mathrm{~h}$ ).

Among the above-described morphologies, the one with droplets is of particular interest due to their potential in many applications such as the generation of nanolenses, concentric plasmonic nanorings or superhydrophobic surfaces. Accordingly, a systematic analysis of their dimension and 3D shape as a function of $h_{B C P}$ was performed. SEM images (Figures 2a-h) show a decrease of the droplets diameter for decreasing $h_{B C P}$. A more precise quantification was performed extracting the diameters $(d)$ from the SEM micrographs with an image processing tool and plotting them as a function of $h_{B C P}$. The results of this analysis, reported in Figure $2 \mathrm{i}$, exhibit a linear decrease of the diameter as $h_{B C P}$ decreases, irrespectively of the RCP. However, the droplet diameter dependence on the $\mathrm{BCP}$ layer thickness is more pronounced for $\mathrm{R} 2$ than $\mathrm{R} 7$ thus suggesting that some interactions of the BCP with the substrate are established in correspondence to the thinnest RCP layer. 

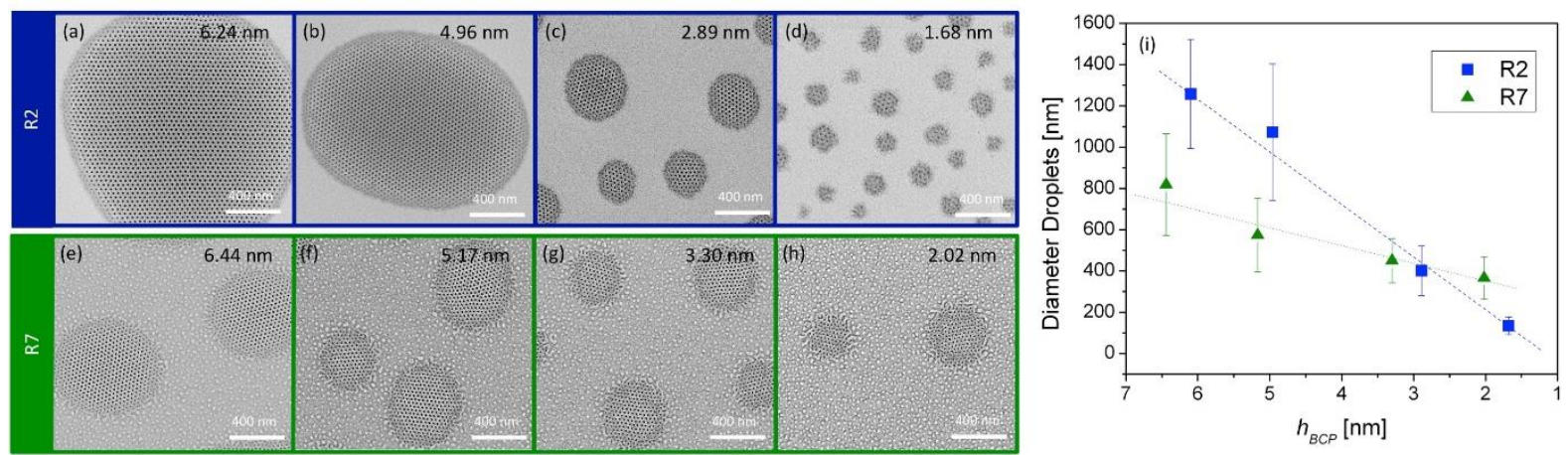

Figure 2: SEM micrographs of the droplets formed on R2 (a)-(d) and on R7 (e)-(h). Diameter of the circular droplets as a function of the BCP thickness (i).

In order to evaluate the 3D shape and the contact angle of the dewetted droplets, atomic force microscopy (AFM) analysis on representative samples was performed. Figure 4 shows AFM maps of BCP samples deposited on R2 (Figures 3a-b) and R7 (Figures 3c-d) and nominal thickness $h_{B C P} \approx 3 \mathrm{~nm}$ before dewetting. These samples were selected in order to compare droplets with similar diameter $(d \approx 400 \mathrm{~nm})$ and distribution. The reported 3D height maps suggest that the shape of the droplets strongly depend on the random copolymer layer thickness. The statistical analysis of the droplet size reveals a linear dependence of droplet height on their diameter for both RCP (Figure 3e), but the dewetted droplets are higher when the $\mathrm{BCP}$ dewets on $\mathrm{R} 2$.
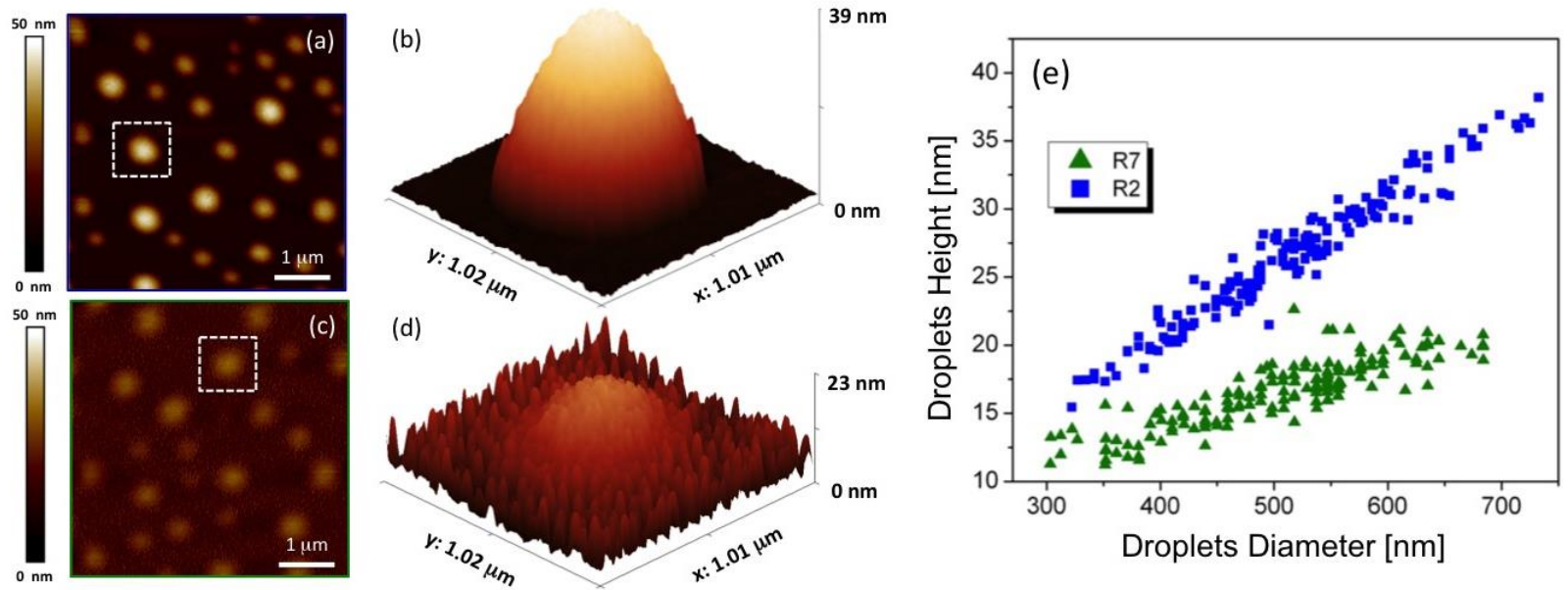

Figure 3: AFM maps and 3D height maps of samples belonging to samples with $h_{B C P} \approx 3$ deposited on R2 (a-b) and R7 (c-d). (e) Height of the droplets formed on R2 and R7 on the same samples as a function its diameter.

The height difference between the droplets dewetted on R2 and R7 reflects the difference in the contact angles that result $\vartheta_{R 2}=11.7^{\circ}$ for R2 and $\vartheta_{R 7}=7.4^{\circ}$ for R7 (see figure S3). In turn, the surface tension $\gamma$ for the BCP deposited on the two RCPs can be estimated from the contact angle values using Young's equation:

$$
{ }_{B R}={ }_{R} \quad{ }_{B} \cos { }_{B R}
$$

where $\gamma_{B R}, \gamma_{B}$ and $\gamma_{R}$ are the surface tensions of the RCP-BCP, BCP-Air and RCP-Air respectively. The values of $\gamma_{B}$ and $\gamma_{R}$ are obtained as the weighted average of the surface tension of PS and PMMA (i.e.: $\gamma_{P S}=$ 
$29.9 \mathrm{erg} / \mathrm{cm}^{2}$ and $\left.\gamma_{P M M A}=30.02 \mathrm{erg} / \mathrm{cm}^{2}\right) .{ }^{55}$ The resulting surface tension for $\mathrm{R} 2$ and $\mathrm{R} 7$ are $\gamma_{R 2}=0.633$ $\mathrm{erg} / \mathrm{cm}^{2}$ and $\gamma_{R 7}=0.261 \mathrm{erg} / \mathrm{cm}^{2}$, respectively. As the composition of R2 and R7 is very similar, the difference in the surface tension is ascribed to the interpenetration of the BCP chains inside the RCP, which extends for approximately $3 \mathrm{~nm} .{ }^{52,67}$ In case of R7, the distance between the BCP layer and the hydrophilic $\mathrm{SiO}_{2}$ surface underneath is higher than the interpenetration depth, thus keeping the BCP layer well isolated from the $\mathrm{SiO}_{2}$ surface. On the other hand, the layer thickness of $\mathrm{R} 2$ is lower than the interpenetration depth. Consequently, some interactions between BCP chains and the polar substrate surface can take place thus ultimately resulting in a higher surface tension.

As a final remark, it is worth to note that the rough profile recorded in the zone surrounding the droplet in Figure $3 \mathrm{~d}$ is not an artifact. AFM micrographs indicate that the surface left uncovered by the BCP after dewetting on R2 and R7 is completely different. While on R2 the surface seems to be perfectly clean and flat (Figures 3a,b), nanometric BCP residues appear on R7, with average diameter of $20 \mathrm{~nm}$ and height $h_{\mathrm{sd}} \approx 7$ $\mathrm{nm}$ (Figures 3c,d). These small droplets are not a peculiarity of the droplet regime, since they can be observed in every morphology once the dewetting is accomplished on R7, as evidenced in Figure 4. These nanometric residues on the clean surface after dewetting appear to have a homogeneous size (from the AFM micrographs) and this is considered compatible with that of an isolated BCP chain laying on $\mathrm{R} 7(\sim 18 \mathrm{~nm})$. Besides the contribution of the contact angle, the presence of isolated BCP chains justifies the lower height of droplets dewetted on R7.

\section{Nanoscale ordering}

In all the experiments presented so far significant differences exist at the micrometric scale between the BCP films dewetting over R2 or R7. However, an accurate analysis of the SEM micrographs revealed that the substrate neutralization also affects the SA process and the ordering at the nanometric scale. To highlight this fact, in Figure 4 the SEM micrographs were overlapped to the color maps representing the angular orientation of grains formed by the hexagonally packed PMMA cylinders embedded in a PS matrix and perpendicularly oriented with respect to the substrate. In correspondence to the borders of the dewetted BCP deposed on R2 (Figures $4 \mathrm{a}-\mathrm{c}$ ), the cylinders form very large grains propagating for several micrometers without any defect. In contrast, when the BCP is deposited on $\mathrm{R} 7$, the formation of grains with smaller dimension and different orientations occurs (Figures 4d-f).

The level of ordering of the self-assembled features can be described in terms of their correlation length $\xi .{ }^{62} \mathrm{In}$ a previous study performed on analogous asymmetric PS- $b$-PMMA treated in RTP at $250{ }^{\circ} \mathrm{C}$, we observed a maximum correlation length $\xi \approx 200 \mathrm{~nm}$. This maximum value was constant in a wide range of BCP thicknesses from 20 to $250 \mathrm{~nm} .{ }^{59}$ However, the introduction of geometric boundaries, as for example periodic trenches, further increases the maximum achievable correlation length. The formation of a single grain propagating along trenches of arbitrary length is observed ${ }^{63}$ when the trench width is smaller than the correlation length of the BCP film deposited on the flat substrate. ${ }^{12}$ However, the correlation length in thin 
BCP films dewetted on R2 is much higher than $200 \mathrm{~nm}$ as can be seen in SEM micrographs reported in Figures $4 a-c$.

Although the origin of the increase in the long-range order in the $\mathrm{BCP}$ dewetted structures on $\mathrm{R} 2$ is not easy to be assessed, the increased nanoscale ordering on R2 seems to be due to the interplay between dewetting and microphase separation surface-polymer interaction energies. Experimental evidences of this mechanism can be found in literature, ${ }^{64,65}$ in which the dewetting process of lamellar-forming BCP directly deposited on the bare substrate without any previous neutralization was investigated by grazing incidence small-angle x-ray scattering (GISAXS) and grazing incidence small-angle neutron scattering (GISANS). The authors concluded that the dewetting process of BCP films can be modeled as a two steps process: in the first step isolated micrometric islands are created via nucleated dewetting, while in the second step the microphase separation process inside these $\mathrm{BCP}$ islands yields to the perpendicularly orientation of the lamellar structure, stabilizing at the same time the dewetted features.

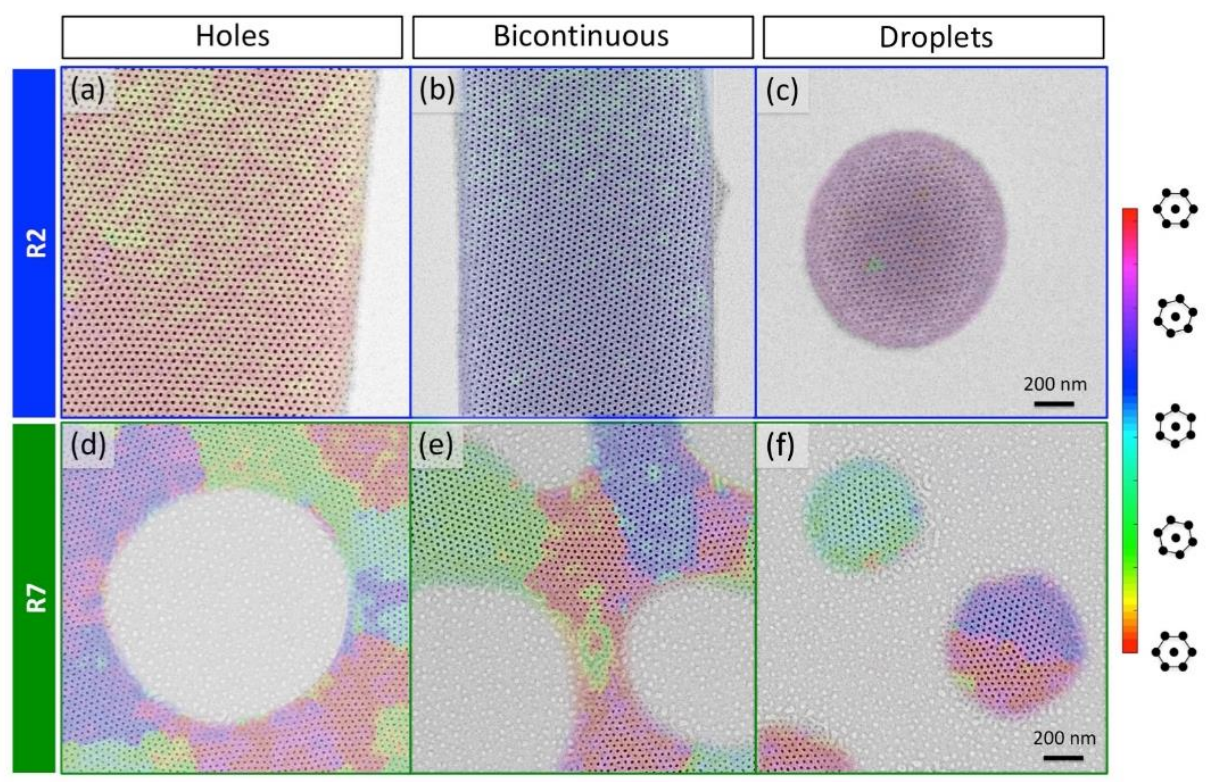

Figure 4: SEM micrographs representing the dewetting front of the BCP on R2 (a-c) and R7 (d-f) in the holes, bicontinuous and droplets regimes.

A more detailed analysis of the dewetting incidence on the degree of lateral order can be obtained from the comparison between the topography of the dewetted features and the grain dimension as illustrated in Figure 5. Here, the AFM height profiles of the dewetting front are reported together with the corresponding SEM micrographs taken in the regimes in which holes (Figures 5a-c) and bicontinuous structures (Figures 5d-f) appear.

The SEM micrograph of Figure 5b is overlapped to the color map representing the grain orientations. In the zone far from the dewetting front, small grains featuring maximum $\xi \approx 156 \mathrm{~nm}$ are present while the grain size gradually increases approaching the dewetting front, up to a value of $\xi \approx 480 \mathrm{~nm}$. This indicates the occurrence of a dewetting-induced ordering in the BCP deposited on R2. This effect propagates for approximately $4 \mu \mathrm{m}$ 
inside the dewetted rim. In this zone, the BCP film thickness (Figure 5c) increases steeply from 10 up to 50 nm.

For the bicontinuous morphology this effect is magnified by the presence of two parallel dewetting fronts, (red arrows in Figure 5d) with the BCP film thickness reaching the value of $\approx 90 \mathrm{~nm}$ (Figure 5e). In this case, the formation of a single grain along the whole dewetted stripe is obtained (Figure 5f).

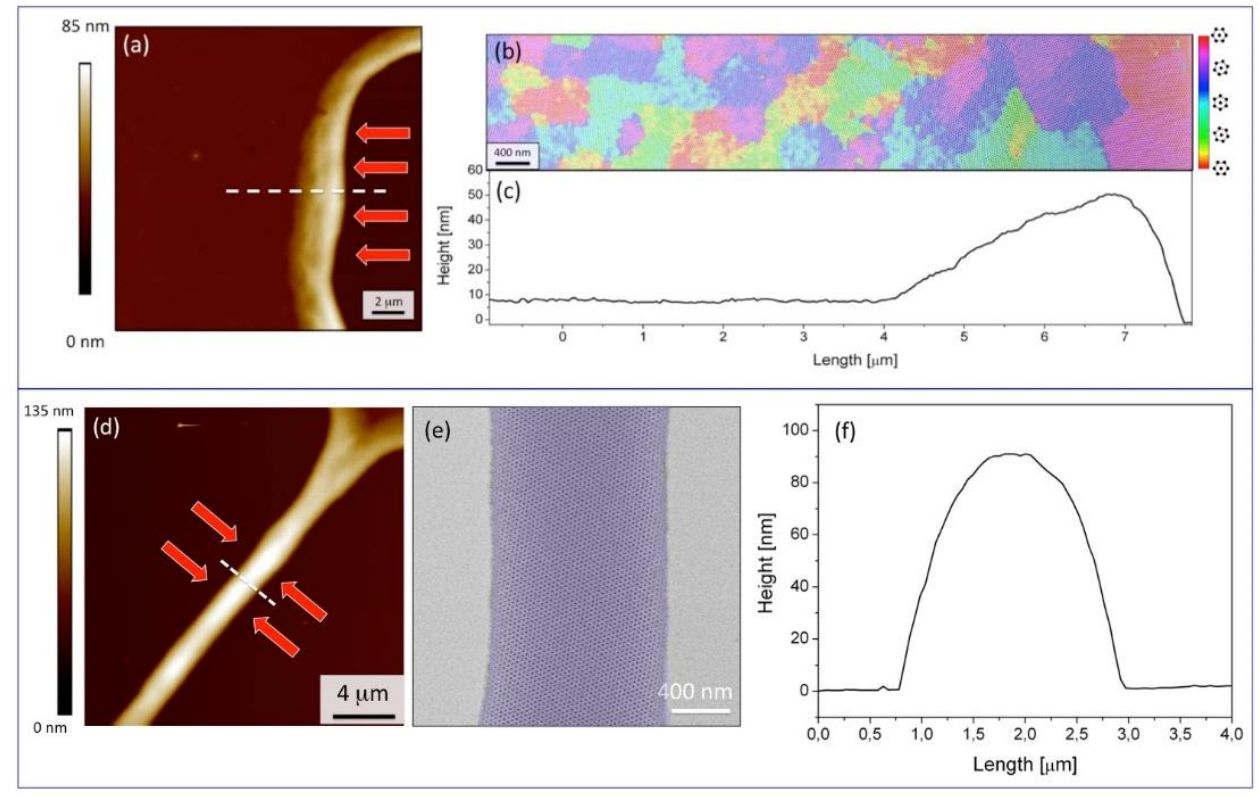

Figure 5: (a) AFM height map acquired in proximity of the side of a hole created by the BCP dewetting on the R2. (b) Color map representing the grain orientation at the edge of the hole and the corresponding height profile (c) extracted along the white dashed line in figure 5a. (d) AFM map, (e) SEM micrograph and (f) height profile obtained in a bicontinuous structure.

\section{Microscale ordering on chemically patterned substrates}

The exploitation of the dewetting process in the production of hierarchically organized structures implies control over the position and size of the dewetted elements. The effect of chemically patterned structures, obtained with periodic micrometric stripes with neutral (RCP) and preferential wettability $\left(\mathrm{SiO}_{2}\right)$ (Figure 6a) is here described. The micrometric pattern was traced by laser writer lithography that does not require complex chemical or lithographic steps, and ensures at the same time the possibility to define micrometric layouts at wafer scale (Figure 6b and S4). Only R2 was employed as surface neutralizer because of the formation of nanometric structures with greater long-range order. 


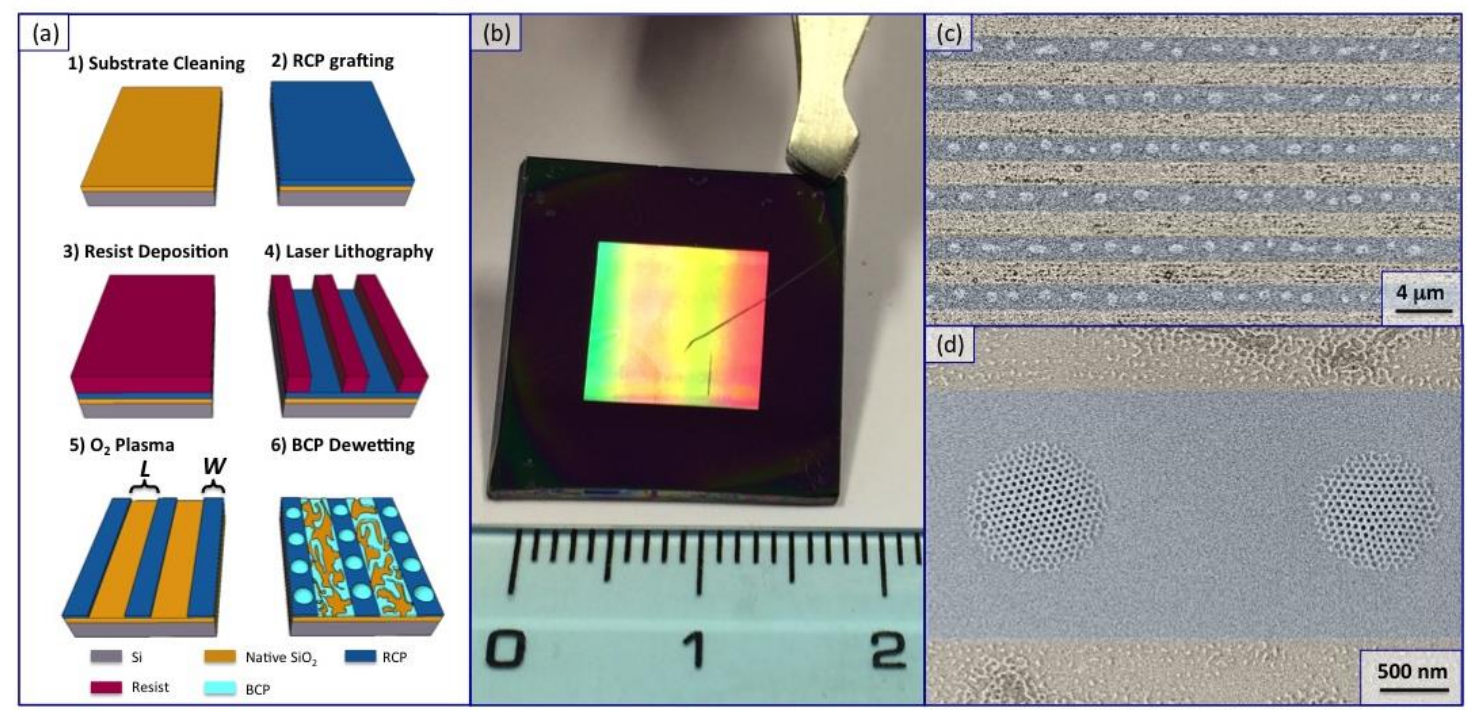

Figure 6: (a) Scheme of the fabrication process of self-assembled droplets on chemically patterned substrates. (b) Large scale image of the periodic pattern after the laser writer lithography step. (c)-(d) SEM micrographs at different magnifications describing the dewetting in chemically modified substrates patterned by laser writer lithography. Colors were included in the SEM images as guide for the eye in order to indicate the self-assembly process on the R 2 and on the native $\mathrm{SiO}_{2}$ (blue and yellow stripes respectively).

Figures $6 \mathrm{c}$ and $6 \mathrm{~d}$ illustrate the effect of dewetting on the patterned substrate at different magnifications. The droplets form exclusively along the neutralized zones (blue stripes in Figure 6c) and are well registered along the stripe direction. On the contrary, over the bare native $\mathrm{SiO}_{2}$ (highlighted in yellow in Figure 6c) pieces of the BCP film consisting of randomly oriented cylinders are observed. In this area, the BCP results highly damaged by the plasma treatment performed during the pore opening procedure.

To better quantify the efficiency of the dewetting process the trend of the average droplet diameter was analyzed as a function of the stripes width $(W)$ (Figure 7). The red band represents the full width at half maximum of the droplets distributed outside the patterned area. These droplets are randomly located on the substrate with a broad Gaussian distribution centered at $d=1260 \mathrm{~nm}$ with a standard deviation of $300 \mathrm{~nm}$ (See Figure S5). Inside the chemical patterns the diameter of the droplets strongly depends on $W$. When $W>$ $3000 \mathrm{~nm}$ the average diameter and distribution of the droplets roughly reproduce those of the unpatterned surface, whereas the formation of multiple rows inside the same stripe is observed (Figure $7 \mathrm{~b}$ ). For $W<3000$ $\mathrm{nm}$, only a single row of droplets is formed on the neutralized area (Figure 7c). The diameter of the droplets decreases linearly from 1200 to $500 \mathrm{~nm}$ when W decreases from 3000 to $1000 \mathrm{~nm}$ and a parallel reduction of the size distribution occurs. 

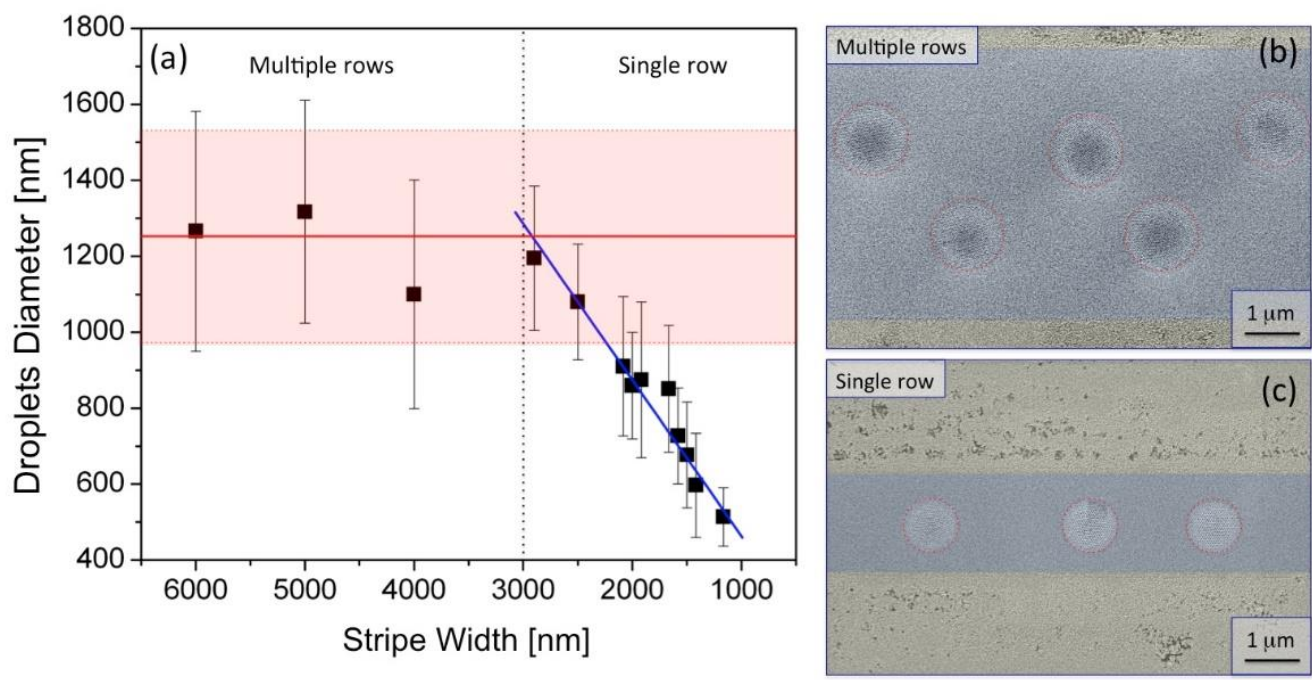

Figure 7: (a) Diameter of the droplets $d$ as a function of the neutralized stripe width $W$. The red zone and indicate the diameter and the distribution of the droplets on the flat not patterned surface. SEM micrographs of representing the droplets (highlighted with red dashed circles) in the multiple rows (b) and single row (c) configurations.

All the droplets formed in the single row consist of circular defect-free grains (Figures 8a-c), though some distortion of the grain lattice can be found in the smallest droplets, due to the irregularity of the edges, as shown by the SEM micrograph in Figure 8d.

Figures $8 \mathrm{e}$ and $8 \mathrm{~g}$ reports the distribution of the droplet diameters formed on chemically patterned stripes with similar $W$ and distance $L$ of 1 (Figure 8e) and $3 \mu \mathrm{m}$ (Figure 8g). The droplet diameter distributions are identical, thus indicating that the line spacing $L$ does not influence the diameter distribution.
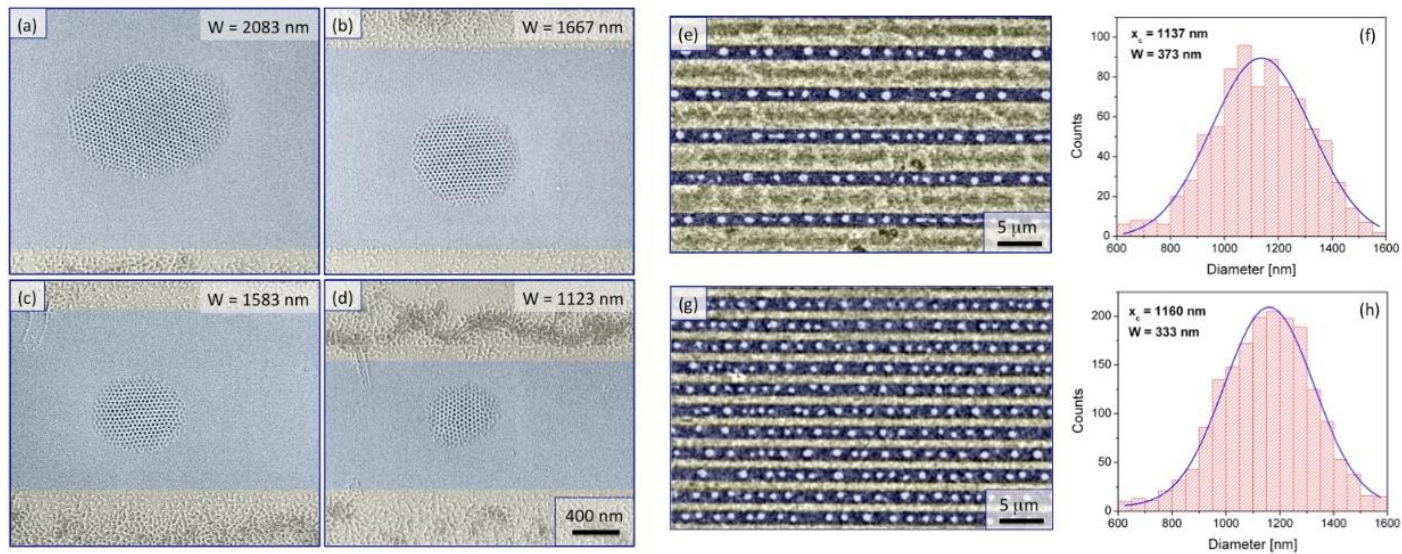

Figure 8: (a)-(d) SEM micrographs of the droplets formed on chemically patterned stripes with decreasing width in the single row configuration. SEM micrographs of chemical patterns with $L=1 \mu \mathrm{m}$ (e) and $3.5 \mu \mathrm{m}$ (g) and nearly identical $W \approx 2.5 \mu \mathrm{m}$.

This observation suggests that in these conditions the droplet formation occurs without flux of BCP from the not neutralized surface towards the neutralized stripe in clear contrast to other directed self-assembled 
systems where BCP flux from the mesa zone ${ }^{66}$ is observed and influences the final thickness of the film confined inside trenches.

\section{CONCLUSIONS}

In conclusion, we investigated the dewetting process of PS-b-PMMA films with thickness $h_{B C P}<10 \mathrm{~nm}$ deposited on a random copolymer grafted layer. Once heated to high temperature $\left(T_{a}=250{ }^{\circ} \mathrm{C}\right)$, PS-bPMMA films dewet, leading to different morphologies including holes in a matrix, bicontinuous structures or circular droplets that are observed to form in sequence when decreasing $h_{B C P}$. The $h_{B C P}$ value corresponding to the ignition of these morphological transitions depends on the thickness of the RCP layer. Moreover, substantial differences are observed in the relevant contact angles $\theta$ of the PS- $b$-PMMA droplets formed on the different RCP grafted layers, with $\theta=11.7^{\circ}$ for the $2 \mathrm{~nm}$ thick RCP layer and $\theta=7.4^{\circ}$ for the $7 \mathrm{~nm}$ thick RCP layer, respectively. Experimental data indicate that the BCP experiences a higher substrate polarity when deposited on the thinner RCP layer, due to a partial interpenetration of the BCP chains extending up to the underlying silicon oxide. The higher contact angle for the $2 \mathrm{~nm}$ thick layer produces also a tight confinement of the BCP droplets that in turn leads to the genesis of circular droplets formed by defectless single grains of self-assembled PS-b-PMMA, with hexagonally packed PMMA cylinders perpendicularly oriented with respect to the substrate. The characteristics of these single grain structures can be further modulated when the BCP dewetting is promoted on chemically patterned stripes of $2 \mathrm{~nm}$ thick RCP films grafted on the substrate. Rows of droplets with micrometer dimension composed of highly ordered hexagonally packed cylinders arranged in a single grain configuration are obtained. Droplet diameter linearly depends on the width of the prepatterned stripes. These results demonstrate the possibility to engineer the dewetting process to fabricate a new class of hierarchical nanostructured materials that could have potential applications in different fields, including nanophotonics or biosensing.

\section{METHODS}

\section{Substrate neutralization}

Silicon substrates, with $\sim 1.6 \mathrm{~nm}$ thick native silicon dioxide $\left(\mathrm{SiO}_{2}\right)$ layer, were used as a support for the BCP SA. The substrates $\left(\sim 2 \mathrm{~cm}^{2}\right.$ surface $)$ were treated with a Piranha solution at $80{ }^{\circ} \mathrm{C}$ for $40 \mathrm{~min}$ to eliminate any residual organic contamination and to increase the surface density of hydroxyl groups. The substrate neutralization was obtained by means of two a-hydroxyl w-Br functional poly(styrene-random-methyl methacrylate) P(S-r-MMA) RCPs with different molecular weight. These RCPs were prepared by activators regenerated by electron transfer atom transfer radical polymerization (ARGET-ATRP) of styrene and methyl methacrylate. ${ }^{67}$ One RCP has $M_{n}=1.69 \mathrm{~kg} / \mathrm{mol}$, styrene fraction $(f)$ of $61.8(\mathrm{wt} / \mathrm{wt})$ and polydispersity index $(\mathrm{PDI})=1.19$ while the other RCP has $M_{n}=14.19 \mathrm{~kg} / \mathrm{mol}, f=61.0$ and PDI $=1.25$. For simplicity in the present manuscript these two RCPs will be labeled respectively as R2 and R7. In both cases a solution with $18 \mathrm{mg}$ of 
$\mathrm{P}(\mathrm{S}-r$-MMA $)$ in $2 \mathrm{ml}$ of toluene was prepared in ultrasonic bath and spun on the substrates at $3000 \mathrm{rpm}$ for 30 s. The grafting process to the $\mathrm{SiO}_{2}$ substrate was performed at high temperature $\left(T_{a}=310{ }^{\circ} \mathrm{C}\right)$ for an annealing time $\left(t_{a}\right)$ of $60 \mathrm{~s} \cdot{ }^{34,68} \mathrm{Afterwards}$, the non-grafted chains were removed by sonication for $300 \mathrm{~s}$ in a toluene bath. The resulting grafted thickness detected by ellipsometry was $2 \mathrm{~nm}$ in the case of R2 and $7.5 \mathrm{~nm}$ for R7.

\section{Block copolymer deposition}

The PS- $b$-PMMA BCP with styrene fraction of $0.71, M_{n}=67.1 \mathrm{~kg} \cdot \mathrm{mol}^{-1}$ and PDI $=1.09$ was purchased from Polymer Source Inc. and used without further purification. The BCP SA process was promoted by annealing the PS- $b$-PMMA films, spun from toluene solutions, at the annealing temperature $\left(T_{a}\right)$ of $250{ }^{\circ} \mathrm{C}$ for $t_{a}=300 \mathrm{~s}$ and a heating rate of $20^{\circ} \mathrm{C} \mathrm{s}^{-1}$. In order to compare the dewetting process of thin BCP films deposited on different RCPs, several samples were prepared featuring different BCP thickness $\left(h_{B C P}\right)$ between 15 and $2 \mathrm{~nm}$. To selectively remove the PMMA from the PS matrix the same pore opening process was performed on all the samples. ${ }^{32}$ The pore opening process consists in exposing the samples to ultraviolet radiation $\left(5 \mathrm{~mW} \cdot \mathrm{cm}^{-2}, \lambda=\right.$ $253.7 \mathrm{~nm}$ ) for $900 \mathrm{~s}$ followed by an immersion in acetic acid bath for $300 \mathrm{~s}$. Finally a isotropic $\mathrm{O}_{2}$ plasma etching was performed at $40 \mathrm{~W}$ for $30 \mathrm{~s}$ in order to remove the residual PMMA at the bottom of the opened pores.

\section{Laser writer lithography}

Chemically patterned substrates were fabricated using a Heidelberg $\mu$ PG101 laser writer, equipped with a 2 $\mathrm{mm}$ laser head $(\lambda=375 \mathrm{~nm})$ producing a laser spot having a maximum resolution of $800 \mathrm{~nm}$.

The commercial optical resist AZ 1505 (Merck Performance Materials GmbH) was spun over the random copolymer R2, previously grafted to the native $\mathrm{SiO}_{2}$ surface. The resist was exposed to a laser spot having beam intensity between 7 and $15 \mathrm{~mW}$. The length of the linear stripes was set to $1 \mathrm{~cm}$ while its width $(W)$ and periodicity $(L)$ were varied from 1 to $10 \mu \mathrm{m}$. A fine-tuning of $W$ between 1 and $2 \mu \mathrm{m}$ was obtained by varying the intensity of the laser beam. After the exposure, the resist was developed for $40 \mathrm{~s}$ in a 1:1 solution of AZ Developer (Merck Performance Materials $\mathrm{GmbH}$ ) and $\mathrm{H}_{2} \mathrm{O}$, and consequently the sample was rinsed in deionized water for $60 \mathrm{~s}$. The RCP in the uncovered zones after the development of the optical resist was removed by exposure to Ar plasma at $40 \mathrm{~W}$ for $120 \mathrm{~s}$. After the removal of the remaining optical resist in acetone, a periodic pattern of neutral and preferential wettability surfaces is obtained. Finally, a $6 \mathrm{~nm}$ BCP film was spin-coated over the chemically patterned substrates and annealed at $250{ }^{\circ} \mathrm{C}$ in RTP.

\section{Film Characterization}

Scanning Electron Microscopy (SEM) analysis (Zeiss Supra 40 SEM) was used to acquire plan view images of the samples in order to evaluate the superficial ordering of the samples. Following the standard procedure described in literature ${ }^{69}$ the correlation length values $\xi_{\text {SEM }}$ were extracted from the SEM micrographs. Atomic Force Microscopy (AFM) imaging was used to trace the 3D shape of the dewetted specimens. Imaging was performed by a Bruker Multimode 8 AFM operating in PeakForce Tapping ${ }^{\circledR}$ with ScanAsyst Air probes (Bruker, U.S.A.). Experiments were performed at room temperature using $\mathrm{J}$ and $\mathrm{E}$ scanners and 
aligning the AFM analysis with the SEM micrographs thanks to the high-magnification camera mounted on the AFM instrument.

The topographical characterizations (in term of diameter, height and contact angle) on the individual dewetted copolymer structures (diameter, height, contact angle) were performed on the AFM micrographs using custom-written software (Matlab, U.S.A.). The measurements were performed semi-automatically: a user points at individual nano/microstructure in a displayed image and an automatic algorithm measures such copolymer structure and displays the results graphically on the image for visual confirmation by the user. This methodology avoids user-induced variability and irreproducibility that commonly affect manual measurements and, at the same time, reduces wrong identification of surface features that fully automated algorithms commonly do in micrographs with a low signal/noise ratio or in correspondence to crowded surfaces. This strategy allows performing measurements of several hundred surface structures, to obtain statistically relevant data with a moderate effort.

\section{ASSICIATED CONTENT}

\section{Supporting Information}

The Supporting Information is available free of charge on the ACS Publications website.

Additional SEM micrographs representing the surface morphology of representative films dewetted on R2 and $\mathrm{R} 7$ for different $\mathrm{BCP}$ thickness, contact angle of the $\mathrm{BCP}$ islands dewetted as a function of the droplet radius, and optical surface profiler 3D height maps of the optical resist after laser writer lithography.

\section{ACKNOWLEDGMENTS}

This research activity has been financially supported by the Project 16ENV07 AEROMET. This project has received funding from the EMPIR programme cofinanced by the EMPIR Participating States and from the European Union's Horizon 2020 Research and Innovation Programme. Part of this work has been performed at Nanofacility INRiM, a laboratory supported by Compagnia di San Paolo Foundation.

\section{REFERENCES}

(1) Wang, L.; Li, Q. Stimuli-Directing Self-Organized 3D Liquid-Crystalline Nanostructures: From Materials Design to Photonic Applications. Adv. Funct. Mater. 2016, 26, 10-28.

(2) Zhang, X.; Lu, W.; Dai, J.; Bourgeois, L.; Yao, J.; Wang, H.; Friend, J. R.; Zhao, D.; MacFarlane, D. R. Nanofabrication of Highly Ordered, Tunable Metallic Mesostructures via Quasi-Hard-Templating of Lyotropic Liquid Crystals. Sci. Rep. 2014, 4, 7420.

(3) Chandramohan, A.; Sibirev, N. V.; Dubrovskii, V. G.; Petty, M. C.; Gallant, A. J.; Zeze, D. A. Model for Large-Area Monolayer Coverage of Polystyrene Nanospheres by Spin Coating. Sci. Rep. 2017, 7, 40888 . 
(4) Chen, K.; Rajeeva, B. B.; Wu, Z.; Rukavina, M.; Dao, T. D.; Al, C. E. T. Moiré Nanosphere Lithography. ACS Nano 2015, 9, 6031-6040.

(5) Chai, J.; Wang, D.; Fan, X. N.; Buriak, J. M. Assembly of Aligned Linear Metallic Patterns on Silicon. Nat. Nanotechnol. 2007, 2, 500-506.

(6) Majewski, P. W.; Rahman, A.; Black, C. T.; Yager, K. G. Arbitrary Lattice Symmetries via Block Copolymer Nanomeshes. Nat. Commun. 2015, 6, 1-6.

(7) Chang, J. B.; Son, J. G.; Hannon, A. F.; Alexander-Katz, A.; Ross, C. A.; Berggren, K. K. Aligned Sub-10-Nm Block Copolymer Patterns Templated by Post Arrays. ACS Nano 2012, 6, 2071-2077.

(8) Suh, H. S.; Kim, D. H.; Moni, P.; Xiong, S.; Ocola, L. E.; Nestor, J.; Gleason, K. K.; Nealey, P. F. Sub-10 Nm Patterning via Directed Self-Assembly of Block Copolymer Films with a Vapour-Phase Deposited Topcoat. Nat. Nanotechnol. 2017, 12, 575-581.

(9) Liu, G.; Thomas, C. S.; Craig, G. S. W.; Nealey, P. F. Integration of Density Multiplication in the Formation of Device-Oriented Structures by Directed Assembly of Block Copolymer-Homopolymer Blends. Adv. Funct. Mater. 2010, 20, 1251-1257.

(10) Tsai, H.; Pitera, J. W.; Miyazoe, H.; Bangsaruntip, S.; Engelmann, S. U.; Liu, C. C.; Cheng, J. Y.; Bucchignano, J. J.; Klaus, D. P.; Joseph, E. A.; Sanders, D. P.; Colburn, M. E.; Guillorn, M. A. TwoDimensional Pattern Formation Using Graphoepitaxy of PS-B-PMMA Block Copolymers for Advanced FinFET Device and Circuit Fabrication. ACS Nano 2014, 8, 5227-5232.

(11) Garner, C. M. Lithography for Enabling Advances in Integrated Circuits and Devices. Philos. Trans. R. Soc. A Math. Phys. Eng. Sci. 2012, 370, 4015-4041.

(12) Aprile, G.; Ferrarese Lupi, F.; Fretto, M.; Enrico, E.; Leo, N. De; Boarino, L.; Volpe, F. G.; Seguini, G.; Sparnacci, K.; Gianotti, V.; Laus, M.; Garnæs, J.; Perego, M. Toward Lateral Length Standards at the Nanoscale Based on Diblock Copolymers. ACS Appl. Mater. Interfaces 2017, 9, 15685-15697.

(13) Hulkkonen, H. H.; Salminen, T.; Niemi, T. Block Copolymer Patterning for Creating Porous Silicon Thin Films with Tunable Refractive Indices. ACS Appl. Mater. Interfaces 2017, 9, 31260-31265.

(14) Dialameh, M.; Ferrarese Lupi, F.; Imbraguglio, D.; Zanenga, F.; Lamperti, A.; Martella, D.; Seguini, G.; Perego, M.; Rossi, A. M.; De Leo, N.; Boarino, L. Influence of Block Copolymer Feature Size on Reactive Ion Etching Pattern Transfer into Silicon. Nanotechnology 2017, 28, 404001.

(15) Frascaroli, J.; Brivio, S.; Ferrarese Lupi, F.; Seguini, G.; Boarino, L.; Perego, M.; Spiga, S. Resistive Switching in High-Density Nanodevices Fabricated by Block Copolymer Self-Assembly. ACS Nano 2015, 9, 2518-2529.

(16) Xiao, S.; Yang, X.; Edwards, E. W.; La, Y.-H.; Nealey, P. F. Graphoepitaxy of Cylinder-Forming Block Copolymers for Use as Templates to Pattern Magnetic Metal Dot Arrays. Nanotechnology 2005, 16, 324-329.

(17) Peng, Q.; Tseng, Y.; Darling, S. B.; Elam, J. W. A Route to Nanoscopic Materials via Sequential Infiltration Synthesis on Block Copolymer Templates. ACS Nano 2011, 5, 4600-4606.

(18) Peng, Q.; Tseng, Y. C.; Darling, S. B.; Elam, J. W. Nanoscopic Patterned Materials with Tunable Dimensions via Atomic Layer Deposition on Block Copolymers. Adv. Mater. 2010, 22, 5129-5133.

(19) Qiu, X.; Yu, H.; Karunakaran, M.; Pradeep, N.; Nunes, S. P.; Peinemann, K. V. Selective Separation of Similarly Sized Proteins with Tunable Nanoporous Block Copolymer Membranes. ACS Nano 2013, 7, 768-776.

(20) Abetz, V. Isoporous Block Copolymer Membranes. Macromol. Rapid Commun. 2015, 36, 10-22. 
(21) Jackson, E. A.; Hillmyer, M. A. Nanoporous Membranes Derived from Block Copolymers: From Drug Delivery to Water Filtration. ACS Nano 2010, 4, 3548-3553.

(22) Dolan, J. A.; Wilts, B. D.; Vignolini, S.; Baumberg, J. J.; Steiner, U.; Wilkinson, T. D. Optical Properties of Gyroid Structured Materials: From Photonic Crystals to Metamaterials. Adv. Opt. Mater. 2015, 3, 12-32.

(23) Song, D.; Li, C.; Li, W.; Watkins, J. J. Block Copolymer Nanocomposites with High Refractive Index Contrast for One-Step Photonics. ACS Nano 2016, 10, 1216-1223.

(24) Mokarian-Tabari, P.; Senthamaraikannan, R.; Glynn, C.; Collins, T. W.; Cummins, C.; Nugent, D.; O’Dwyer, C.; Morris, M. A. Large Block Copolymer Self-Assembly for Fabrication of Subwavelength Nanostructures for Applications in Optics. Nano Lett. 2017, 17, 2973-2978.

(25) Rahman, A.; Majewski, P. W.; Doerk, G.; Black, C. T.; Yager, K. G. Non-Native Three-Dimensional Block Copolymer Morphologies. Nat. Commun. 2016, 7, 1-8.

(26) Palmer, J. S.; Swaminathan, P.; Babar, S.; Weaver, J. H. Solid-State Dewetting-Mediated Aggregation of Nanoparticles. Phys. Rev. B - Condens. Matter Mater. Phys. 2008, 77, 1-10.

(27) Roy, S.; Bandyopadhyay, D.; Karim, A.; Mukherjee, R. Interplay of Substrate Surface Energy and Nanoparticle Concentration in Suppressing Polymer Thin Film Dewetting. Macromolecules 2015, 48, 373-382.

(28) Geldmeier, J.; Rile, L.; Yoon, Y. J.; Jung, J.; Lin, Z.; Tsukruk, V. V. Dewetting-Induced Photoluminescent Enhancement of Poly(lauryl methacrylate)/Quantum Dot Thin Films. Langmuir 2017, 33, 14325-14331.

(29) Zhang, X.; Ren, J.; Yang, H.; He, Y.; Tan, J.; Qiao, G. G. From Transient Nanodroplets to Permanent Nanolenses. Soft Matter 2012, 8, 4314.

(30) Verma, A.; Sharma, A. Self-Organized Nano-Lens Arrays by Intensified Dewetting of Electron Beam Modified Polymer Thin-Films. Soft Matter 2011, 7, 11119-11124.

(31) Lal, J.; Malkova, S.; Mukhopadhyay, M. K.; Narayanan, S.; Fluerasu, A.; Darling, S. B.; Lurio, L. B.; Sutton, M. Dewetting in Immiscible Polymer Bilayer Films. Phys. Rev. Mater. 2017, 1, 15601.

(32) Müller-Buschbaum, P.; Bauer, E.; Wunnicke, O.; Stamm, M. The Control of Thin Film Morphology by the Interplay of Dewetting, Phase Separation and Microphase Separation. J. Phys. Condens. Matter 2005, 17, S363-S386.

(33) Seemann, R.; Herminghaus, S.; Jacobs, K. Dewetting Patterns and Molecular Forces : A Reconciliation. Phys. Rev. Lett. 2001, 86, 5534-5537.

(34) Peng, J.; Xing, R.; Wu, Y.; Li, B.; Han, Y.; Knoll, W.; Kim, D. H. Dewetting of Thin Polystyrene Films under Confinement. Langmuir 2007, 23, 2326-2329.

(35) Cai, X.; Genzer, J.; Spontak, R. J. Evolution of Homopolymer Thin-Film Instability on SurfaceAnchored Diblock Copolymers Varying in Composition. Langmuir 2014, 30, 11689-11695.

(36) Ramanathan, M.; Darling, S. B. Mesoscale Morphologies in Polymer Thin Films. Prog. Polym. Sci. 2011, 36, 793-812.

(37) Bhandaru, N.; Das, A.; Salunke, N.; Mukherjee, R. Ordered Alternating Binary Polymer Nanodroplet Array by Sequential Spin Dewetting. Nano Lett. 2014, 14, 7009-7016.

(38) Roy, S.; Biswas, D.; Salunke, N.; Das, A.; Vutukuri, P.; Singh, R.; Mukherjee, R. Control of Morphology in Pattern Directed Dewetting of a Thin Polymer Bilayer. Macromolecules 2013, 46, 935-948. 
(39) Baek, K. M.; Kim, J. M.; Jeong, J. W.; Lee, S. Y.; Jung, Y. S. Sequentially Self-Assembled Rings-inMesh Nanoplasmonic Arrays for Surface-Enhanced Raman Spectroscopy. Chem. Mater. 2015, 27, 5007-5013.

(40) Robbins, S. W.; Beaucage, P. A.; Sai, H.; Tan, K. W.; Werner, J. G.; Sethna, J. P.; Disalvo, F. J.; Gruner, S. M.; Dover, R. B. Van; Wiesner, U. Block Copolymer Self-Assembly-directed Synthesis of Mesoporous Gyroidal Superconductors. Sci. Adv. 2016, 2, 1-8.

(41) Stefik, M.; Guldin, S.; Vignolini, S.; Wiesner, U.; Steiner, U. Block Copolymer Self-Assembly for Nanophotonics. Chem. Soc. Rev. 2015, 44, 5076-5091.

(42) Park, S.; Cheng, X.; Böker, A.; Tsarkova, L. Hierarchical Manipulation of Block Copolymer Patterns on 3D Topographic Substrates : Beyond Graphoepitaxy. Adv. Mater. 2016, 28, 6900-6905.

(43) Qiu, H.; Hudson, Z. M.; Winnik, M. A.; Manners, I. Multidimensional Hierarchical Self-Assembly of Amphiphilic Cylindrical Block Comicelles. Science 2015, 347, 1329-1332.

(44) Sun, Y. S.; Chien, S. W.; Wu, P. J. Effects of Film Instability on Roughness Correlation and Nanodomain Ordering in Ultrathin Films of Asymmetric Block Copolymers. Macromolecules 2010, $43,5016-5023$.

(45) Yan, D.; Huang, H.; He, T.; Zhang, F. Coupling of Microphase Separation and Dewetting in Weakly Segregated Diblock Co-Polymer Ultrathin Films. Langmuir 2011, 27, 11973-11980.

(46) Limary, R.; Green, P. F. Dewetting Instabilities in Thin Block Copolymer Films: Nucleation and Growth. Langmuir 1999, 15, 5617-5622.

(47) Masson, J.-L.; Limary, R.; Green, P. F. Pattern Formation and Evolution in Diblock Copolymer Thin Films above the Order-disorder Transition. J. Chem. Phys. 2001, 114, 10963.

(48) Ceresoli, M.; Ferrarese Lupi, F.; Seguini, G.; Sparnacci, K.; Gianotti, V.; Antonioli, D.; Laus, M.; Boarino, L.; Perego, M. Evolution of Lateral Ordering in Symmetric Block Copolymer Thin Films upon Rapid Thermal Processing. Nanotechnology 2014, 25, 275601.

(49) Farrell, R. A.; Kehagias, N.; Shaw, M. T.; Reboud, V.; Zelsmann, M.; Holmes, J. D.; Sotomayor Torres, C. M.; Morris, M. A. Surface-Directed Dewetting of a Block Copolymer for Fabricating Highly Uniform Nanostructured Microdroplets and Concentric Nanorings. ACS Nano 2011, 5, 1073 1085 .

(50) Onses, M. S.; Song, C.; Williamson, L.; Sutanto, E.; Ferreira, P. M.; Alleyne, A. G.; Nealey, P. F.; Ahn, H.; Rogers, J. A. Hierarchical Patterns of Three-Dimensional Block-Copolymer Films Formed by Electrohydrodynamic Jet Printing and Self-Assembly. Nat. Nanotechnol. 2013, 8, 667-675.

(51) Hur, S.; Onses, M. S.; Ram, A.; Nealey, P. F.; Rogers, J. A.; Pablo, J. J. De. Interplay of Surface Energy and Bulk Thermodynamic Forces in Ordered Block Copolymer Droplets. Macromolecules 2015, 48, 4717-4723.

(52) Ryu, D. Y.; Shin, K.; Drockenmuller, E.; Hawker, C. J.; Russel, T. P. A Generalized Approach to the Modifiction of Solid Surfaces. Science 2004, 308, 236-238.

(53) Ceresoli, M.; Palermo, M.; Ferrarese Lupi, F.; Seguini, G.; Perego, M.; Zuccheri, G.; Phadatare, S. D.; Antonioli, D.; Gianotti, V.; Sparnacci, K.; Laus, M. Neutral Wetting Brush Layers for Block Copolymer Thin Films Using Homopolymer Blends Processed at High Temperatures. Nanotechnology 2015, 26, 415603.

(54) Epps, T. H.; Delongchamp, D. M.; Fasolka, M. J.; Fischer, D. A.; Jablonski, E. L.; Engineering, C.; V, B. U.; Pennsyl, V. Substrate Surface Energy Dependent Morphology and Dewetting in an ABC Triblock Copolymer Film. Langmuir 2007, 32, 3355-3362. 
(55) Mansky, P.; Liu, Y.; Russell, T. P.; Hawker, C. Controlling Polymer-Surface Interactions with Random Copolymer Brushes. Science 1997, 275, 1458-1460.

(56) Sparnacci, K.; Antonioli, D.; Gianotti, V.; Laus, M.; Zuccheri, G.; Ferrarese Lupi, F.; Giammaria, T. J.; Seguini, G.; Ceresoli, M.; Perego, M. Thermal Stability of Functional P(S-R-MMA) Random Copolymers for Nanolithographic Applications. ACS Appl. Mater. Interfaces 2015, 7, 3920-3930.

(57) Ji, S.; Liu, C.-C.; Son, J. G.; Gotrik, K.; Craig, G. S. W.; Gopalan, P.; Himpsel, F. J.; Char, K.; Nealey, P. F. Generalization of the Use of Random Copolymers To Control the Wetting Behavior of Block Copolymer Films. Macromolecules 2008, 41, 9098-9103.

(58) Giammaria, T. J.; Ferrarese Lupi, F.; Seguini, G.; Sparnacci, K.; Antonioli, D.; Gianotti, V.; Laus, M.; Perego, M.; Mdm, L.; Olivetti, V. C.; Brianza, A. Effect of Entrapped Solvent on the Evolution of Lateral Order in Self- Assembled P(S-r-MMA)/PS-b-PMMA Systems with Different Thicknesses. Appl. Mater. interfaces 2017, 9, 31215-31223.

(59) Ferrarese Lupi, F.; Giammaria, T. J.; Volpe, F. G.; Lotto, F.; Seguini, G.; Pivac, B.; Laus, M.; Perego, M. High Aspect Ratio PS-b-PMMA Block Copolymer Masks for Lithographic Applications. ACS Appl. Mater. Interfaces 2014, 6, 21389-21396.

(60) Nečas, D.; Klapetek, P. Gwyddion: An Open-Source Software for SPM Data Analysis. Cent. Eur. J. Phys. 2012, 10.

(61) Green, P. F.; Limary, R. Block Copolymer Thin Films : Pattern Formation and Phase Behavior. Adv. Colloid Interface Sci. 2001, 94, 53-81.

(62) Doerk, G. S.; Yager, K. G. Rapid Ordering in "Wet Brush" Block Copolymer/Homopolymer Ternary Blends. ACS Nano 2017, 11, 12326-12336.

(63) Perego, M.; Andreozzi, A.; Vellei, A.; Ferrarese Lupi, F.; Seguini, G. Collective Behavior of Block Copolymer Thin Films within Periodic Topographical Structures. Nanotechnology 2013, 24, 245301.

(64) Müller-Buschbaum, P.; Hermsdorf, N.; Roth, S. V.; Wiedersich, J.; Cunis, S.; Gehrke, R. Comparative Analysis of Nanostructured Diblock Copolymer Films. Spectrochim. Acta - Part B At. Spectrosc. 2004, 59, 1789-1797.

(65) Müller-Buschbaum, P.; Cubitt, R.; Petry, W. Nanostructured Diblock Copolymer Films: A Grazing Incidence Small-Angle Neutron Scattering Study. Langmuir 2003, 19, 7778-7782.

(66) Ferrarese Lupi, F.; Aprile, G.; Giammaria, T. J.; Seguini, G.; Zuccheri, G.; De Leo, N.; Boarino, L.; Laus, M.; Perego, M. Thickness and Microdomain Orientation of Asymmetric PS-b-PMMA Block Copolymer Films Inside Periodic Gratings. ACS Appl. Mater. Interfaces 2015, 7, 23615-23622.

(67) Sparnacci, K.; Antonioli, D.; Gianotti, V.; Laus, M.; Ferrarese Lupi, F.; Giammaria, T. J.; Seguini, G.; Perego, M. Ultrathin Random Copolymer-Grafted Layers for Block Copolymer Self-Assembly. ACS Appl. Mater. Interfaces 2015, 7, 10944-10951.

(68) Sparnacci, K.; Antonioli, D.; Perego, M.; Giammaria, T. J.; Seguini, G.; Ferrarese Lupi, F.; Zuccheri, G.; Gianotti, V.; Laus, M. High Temperature Surface Neutralization Process with Random Copolymers for Block Copolymer Self-Assembly. Polym. Int. 2017, 66, 459-467.

(69) Ji, S.; Liu, C.; Liao, W.; Fenske, A. L.; Craig, G. S. W.; Nealey, P. F. Domain Orientation and Grain Coarsening in Cylinder-Forming Poly (Styrene-B-Methyl Methacrylate) Films. Macromolecules 2011, 44, 4291-4300. 